\title{
Adaptable Multivariate Calibration Models for Spectral Applications
}

\author{
Edward V. Thomas
}

Sandia National Laboratories

Albuquerque, NM 87185-0829

\author{
Abstract \\ DEC 271999 \\ Multivariate calibration techniques have been used in a wide variety of spectroscopic \\ situations. In many of these situations spectral variation can be partitioned into \\ meaningful classes. For example, suppose that multiple spectra are obtained from each \\ of a number of different objects wherein the level of the analyte of interest varies within \\ each object over time. In such situations the total spectral variation observed across all \\ measurements has two distinct general sources of variation: intra-object and inter-object. \\ One might want to develop a global multivariate calibration model that predicts the \\ analyte of interest accurately both within and across objects, including new objects not \\ involved in developing the calibration model. However, this goal might be hard to \\ realize if the inter-object spectral variation is complex and difficult to model. If the intra- \\ object spectral variation is consistent across objects, an effective alternative approach \\ might be to develop a generic intra-object model that can be adapted to each object \\ separately. This paper contains recommendations for experimental protocols and data \\ analysis in such situations. The approach is illustrated with an example involving the \\ noninvasive measurement of glucose using near-infrared reflectance spectroscopy. \\ Extensions to calibration maintenance and calibration transfer are discussed.
}




\section{DISCLAIMER}

This report was prepared as an account of work sponsored by an agency of the United States Government. Neither the United States Government nor any agency thereof, nor any of their employees, make any warranty, express or implied, or assumes any legal liability or responsibility for the accuracy, completeness, or usefulness of any information, apparatus, product, or process disclosed, or represents that its use would not infringe privately owned rights. Reference herein to any specific commercial product, process, or service by trade name, trademark, manufacturer, or otherwise does not necessarily constitute or imply its endorsement, recommendation, or favoring by the United States Government or any agency thereof. The views and opinions of authors expressed herein do not necessarily state or reflect those of the United States Government or any agency thereof. 


\section{DISCLAIMER}

Portions of this document may be illegible in electronic image products. Images are produced from the best available original document. 


\section{Introduction}

Multivariate calibration has received significant attention in analytical chemistry, particularly in spectroscopy. Martens and Naes ${ }^{1}$ provide an excellent general reference on multivariate calibration. Examples of multivariate calibration in a spectroscopic context are associated with applications in the agricultural and food industries ${ }^{2}$, manufacturing industries ${ }^{3}$, medical sciences ${ }^{4}$, the pharmaceutical industries ${ }^{5}$, and in many other contexts. See the review by Lavine ${ }^{6}$ for more examples.

The development of a multivariate calibration model in spectroscopy involves the measurement of $n$ specimens at $q$ different wavelengths comprising the calibration set. In the case of a linear multivariate calibration model, the resultant predictive model can be expressed in the form,

$$
\hat{y}=b_{0}+b_{1} \cdot x_{1}+b_{2} \cdot x_{2}+\ldots+b_{q} \cdot x_{q},
$$

where $\hat{y}$ is the predicted value of the analyte of interest for a new specimen given the spectral measurements $\left(x_{i}{ }^{\prime} s\right)$ associated with the new specimen and the model parameters $\left(b_{i}\right.$ 's). Common methods for obtaining the model parameters include partial least-squares regression (PLS) and principal components regression (PCR) ${ }^{1}$. The efficacy of the predictive model depends on the applicability of the model form and how well the calibration set represents the compositions of the new specimens on which the predictive model will be applied. The efficacy of the predictive model also depends on how well the calibration set spans instrumental/environmental conditions expected in the future. This is particularly critical when spectral effects induced by instrumental variation (e.g., source fluctuations) and the instrument's local environment (e.g., temperature) are nontrivial when compared to effects introduced by varying the compositional factors ${ }^{7}$. 
In many situations, it would be desirable to apply the predictive model to spectra obtained across and within different objects. It is assumed that the objects are dynamic in the sense that the level of the analyte of interest can vary from spectrum to spectrum within an object. In such situations, the nature of the spectral variation across objects might make it difficult to develop a single model of the form in Equation 1 that is valid across objects. However, if the intra-object spectral variation is similar across objects and amenable to a model of the form in Equation 1, there is a viable alternative approach. This approach, which is the topic of this paper, is to develop a generic model (operable within an object) that can be adapted to each object separately. This paper contains recommendations for experimental protocols and data analysis in such situations. The approach is illustrated with an example involving the noninvasive measurement of glucose using near-infrared reflectance spectroscopy where the objects are different individuals. Extensions of this approach to calibration maintenance and transfer are discussed.

\section{Theory}

Consider $q$-dimensional spectral measurements that are obtained within and across a population of objects. Let $x_{i j}=\left[x_{i j 1}, x_{i j 2}, \ldots, x_{i j q}\right]$ denote the $j^{\text {th }}$ spectrum associated with the $i^{\text {th }}$ object. This spectrum can be represented by

$$
x_{i j}=\mu+\alpha_{i}+\varepsilon_{i j}
$$

where $\mu=\left[\mu_{1}, \mu_{2}, \ldots, \mu_{q}\right]$ is the average spectrum across the population, $\alpha_{i}=\left[\alpha_{i 1}, \alpha_{i 2}, \ldots, \alpha_{i q}\right]$ is the specific effect of the $i^{t h}$ object on the spectrum, and $\varepsilon_{i j}=\left[\varepsilon_{i 1}, \varepsilon_{i 2}, \ldots, \varepsilon_{i q}\right]$ is the specific effect of the $j^{\text {th }}$ spectrum of the $i^{t h}$ object. It is 
assumed that the $\varepsilon_{i j}$ term describes random spectral effects that, in a sense, have a common distribution across objects. That is, these effects are just as likely to be associated with one object as well as another. In contrast, the $\boldsymbol{\alpha}_{i}$ effects are specific to an object. It is also assumed that the level of the associated analyte of interest varies across the measurements of each object (e.g., the analyte of interest could be changing in time). Furthermore it is assumed that a unit-change in the analyte of interest induces an identical spectral effect across objects.

The usual strategy for developing a global model (one that is applicable across objects) is to use a modeling method such as PLS or PCR in conjunction with an appropriate calibration set containing spectra from multiple objects. The calibration set consists of $X$ and $Y$, where $X=\left[X_{1}, X_{2}, \ldots, X_{\mathrm{I}}\right], Y=\left[y_{1}, y_{2}, \ldots, y_{\mathrm{I}}\right], X_{i}=\left[x_{i 1}, x_{i 2}, \ldots, x_{i n_{i}}\right]$ is the $n_{i} x q$ matrix (i.e. number of spectra by number of pixels) of the calibration spectra from the $\mathrm{i}^{\text {th }}$ of I objects, and $y_{i}=\left[\mathrm{y}_{i l}, y_{i 2}, \ldots, y_{i i_{i}}\right]$ is the $n_{i} x 1$ vector of the associated analyte reference values. The dimensions of $\boldsymbol{X}$ and $\boldsymbol{Y}$ are $N x q$ and $N x 1$ respectively, where $N=\sum_{i=1}^{I} n_{i}$. A linear calibration model is obtained by using a method such as PLS (or PCR) with $\boldsymbol{X}$ and $\boldsymbol{Y}$. The resulting predictor of the analyte level associated with the $j^{t h}$ spectrum of the $i^{\text {th }}$ object (denoted by $y_{i j}$ ) can be expressed as

$$
\hat{y}_{i j}=b_{0}+\sum_{k=1}^{q} b_{k} \cdot x_{i j k}
$$

The set of coefficients $\left\{b_{k}\right\}_{k=1, \ldots, q}$ are sometimes referred to as final regression coefficients. Note that Equation 3 can be expanded (via Equation 2) as

$$
\hat{y}_{i j}=b_{0}+\sum_{k=1}^{q} b_{k} \cdot\left(\mu_{k}+\alpha_{i k}+\varepsilon_{i j k}\right) \text {. }
$$


In the usual strategy for developing a global model, the inter-object spectral effects and intra-object spectral effects are not considered independently. That is, the model is developed in view of the combined inter- and intra-object effects $\left(\boldsymbol{\alpha}_{i}\right.$ and $\left.\varepsilon_{i j}\right)$. As long as both the inter- and intra-object effects are amenable to modeling, this strategy can be effective. On the other hand, if the inter-object spectral effects are for some reason difficult to model (e.g., the calibration data do not sufficiently span the inter-object effects), then this approach may be ineffective.

An alternative approach involves modeling the intra-object spectral effects separately and then adapting the model to a target object. In many cases, the intra-object effects are small relative to the inter-object effects and are distributed similarly across objects. The latter condition is assumed here. In this approach the calibration data are mean-centered by object. That is $\boldsymbol{X}^{m c}=\left[\boldsymbol{X}_{1}^{m c}, \boldsymbol{X}_{2}^{m c}, \ldots, \boldsymbol{X}_{I}^{m c}\right]$ and

$$
\begin{gathered}
\boldsymbol{Y}^{m c}=\left[\boldsymbol{y}_{1}^{m c}, \boldsymbol{y}_{2}^{m c}, \ldots, \boldsymbol{y}_{I}^{m c}\right], \text { where } \\
\boldsymbol{X}_{i}^{m c}=\left[\boldsymbol{x}_{i 1}^{m c}, \boldsymbol{x}_{i 2}^{m c}, \ldots, \boldsymbol{x}_{i i_{i}}^{m c}\right]=\left[\boldsymbol{x}_{i 1}-\overline{\boldsymbol{x}}_{i}, \boldsymbol{x}_{i 2}-\overline{\boldsymbol{x}}_{i}, \ldots, \boldsymbol{x}_{i n_{i}}-\overline{\boldsymbol{x}}_{i}\right] \\
\boldsymbol{y}_{i}^{m c}=\left[y_{i 1}-\bar{y}_{i}, y_{i 2}-\bar{y}_{i}, \ldots, y_{i i_{i}}-\bar{y}_{i}\right], \\
\overline{\boldsymbol{x}}_{i}=\frac{1}{n_{i}} \cdot \sum_{j=1}^{n_{i}} \boldsymbol{x}_{i j}, \text { and } \bar{y}_{i}=\frac{1}{n_{i}} \cdot \sum_{j=1}^{n_{i}} y_{i j} . \text { The mean-centering operation on the spectra }
\end{gathered}
$$

removes the average spectrum $(\mu)$ and the object-specific spectral effect $\left(\alpha_{i}\right)$ from the original calibration spectra leaving only the specific effect of the $j^{\text {th }}$ spectrum of the $i^{\text {th }}$ object (see Equation 1). That is

$$
x_{i j}^{m c}=\varepsilon_{i j} .
$$

$\boldsymbol{X}^{m c}$ and $\boldsymbol{Y}^{m c}$ will heretofore be referred to as generic calibration data. 
Standard multivariate calibration techniques like PLS and PCR can be applied to the generic calibration data to develop a calibration model that relates variation of the analyte of interest to intra-object spectral effects. Note that the mean-centering operations ensure that both $\boldsymbol{X}^{m c}$ and $\boldsymbol{Y}^{m c}$ have a mean of zero. Thus it is unnecessary to include an intercept in these modeling activities, resulting in a predictive model of the form,

$$
\hat{y}_{i j}^{m c}=\sum_{k=1}^{q} g_{k} \cdot \varepsilon_{i j k}
$$

This model is referred to as a generic model as it is applicable to intra-object spectral variation from all objects. The model coefficients $\left(\left\{g_{k}\right\}_{\mathrm{k}=1, \ldots, \mathrm{q}}\right)$ are differentiated from those in Equation 3. Because the generic model is developed with regard to intra-object spectral variation only, these model coefficients will not generally be the same as those associated with the global model $\left(\left\{b_{k}\right\}_{\mathrm{k}=1, \ldots, \mathrm{q}}\right)$. Note that the quality of the model depends on the level of the intra-object variation of the analyte of interest (i.e. the variation in $\boldsymbol{Y}^{m c}$ ). For maximum benefit, it is important to observe each object in the calibration set at a relatively wide range of analyte levels.

In order to be a viable predictor of the analyte of interest, the generic model must be adapted to each new target object. The global model (in Equation 4) does this adaptation implicitly through the fitted model coefficients that are developed in recognition of inter-object spectral effects as well as the intra-object spectral effects. In Equation $4, \sum_{k=1}^{q} b_{k} \cdot \mu_{k}$ is a constant that does not depend on $i$ or $j$. Furthermore, $\sum_{k=1}^{q} b_{k} \cdot \alpha_{i k}$ is an object-specific constant. Thus, Equation 4 can be rewritten as 


$$
\hat{y}_{i j}=d_{i}+\sum_{k=1}^{q} b_{k} \cdot \varepsilon_{i j k}
$$

Cast in this formulation, it is clear that $d_{i}=\sum_{k=1}^{q} b_{k} \cdot \alpha_{i k}$ provides an adaptation to the $i^{t h}$ object through the interaction of the regression coefficients $\left(\left\{b_{k}\right\}\right)$ and the object-specific spectral effects $\left(\alpha_{i}\right)$. Hence the adaptation is linear with respect to the object-specific spectral effects.

In the case of the generic model, the proposed adaptation takes a similar form. That is, for the $p^{\text {th }}$ prediction sample associated with the $t^{\text {th }}$ target object (where the $t^{\text {th }}$ target object is not normally represented in the calibration set), we seek to find an appropriate value of $c_{t}$ such that

$$
\hat{y}_{t p}=c_{t}+\sum_{k=1}^{q} g_{k} \cdot \varepsilon_{t p k}
$$

is a good predictor of $y_{t p}$. Equivalently, we want to find a value of $f_{t}$ such that

$$
\hat{y}_{t p}=f_{t}+\sum_{k=1}^{q} g_{k} \cdot x_{t p k}
$$

is a good predictor of $y_{t p}$ given the spectral measurement, $x_{t p k}$. A direct way to accomplish this is to use one or more representative spectra and associated reference

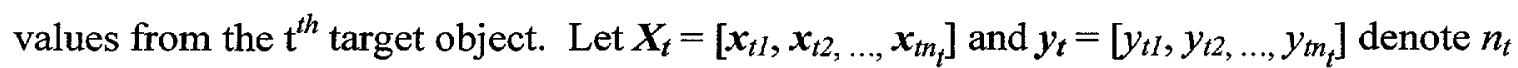
representative spectra and associated reference values from the $\mathrm{t}^{\text {th }}$ target object. Adaptation to the $t^{\text {th }}$ target object is provided by

$$
f_{t}=\sum_{j=1}^{n_{t}} y_{i j}-\sum_{j=1}^{n_{t}} \sum_{k=1}^{q} g_{k} \cdot x_{t j k}
$$

The predictor based on $f_{t}$ given in Equation 12 is constructed so that, over the $n_{t}$ 
representative spectra, the average prediction will equal the average of the associated reference analyte values. It is important to emphasize that this method of adaptation depends on an accurate reference value for each representative spectrum that is used to adapt. Inaccuracies in the reference analyte values will adversely affect the quality of the adaptation.

An important benefit of the generic modeling approach is the potential for the detection of outliers in prediction that is focused entirely on intra-object spectral effects. In the case of the development of global models, outlier detection metrics are likely to be based heavily on inter-object effects and, therefore, not be sufficiently responsive to unusual intra-object effects. In the generic modeling approach, inter-object effects are ancillary. While outlier detection metrics in the generic modeling approach can be very effective for identifying anomalous spectra, some care is required in their construction.

In order to illustrate how outlier metrics can be constructed in the generic modeling context, let $\boldsymbol{d}_{t p}=\boldsymbol{x}_{t p}-\operatorname{mean}\left(\boldsymbol{X}_{t}\right)$ represent the deviation of the $p^{\text {th }}$ prediction spectrum from the average of the associated $n_{t}$ adaptation spectra with all spectra associated with the target object. Note that the elements of $\boldsymbol{d}_{t p}$ can be rewritten as

$$
d_{t p k}=\varepsilon_{t p k}-\operatorname{mean}\left(\varepsilon_{t j k}\right),
$$

since the object-specific spectral effects are removed from $\boldsymbol{x}_{t p}$ via the subtraction of mean $\left(\boldsymbol{X}_{\boldsymbol{t}}\right)$. In the sense that object-specific spectral effects have been removed, $\boldsymbol{d}_{t}$ conforms to the modeling space given by $\boldsymbol{X}^{m c}$. To continue, let the integrated unmodelled residual of the $\mathrm{p}^{\text {th }}$ spectrum from the target object be defined by,

$$
R_{t p}=\boldsymbol{e}_{t p}{ }^{T} \boldsymbol{e}_{t p},
$$

where $\boldsymbol{e}_{t p}$ is the unmodelled portion of $\boldsymbol{d}_{t p}$ (see e.g., p. 291 in Martens and Naes ${ }^{1}$ ). 
Normally, a prediction spectrum is deemed anomalous if its integrated unmodelled residual is unusually large when compared to the distribution of similarly computed values from the calibration set. Here, however, due to the use of the adaptation spectra, one should compare $S_{t p}=\frac{n_{t}}{n_{t}+1} \cdot R_{t p}$ with the distribution of $R_{i j}$ 's that are derived from the calibration set. The normalizing factor $\frac{n_{t}}{n_{t}+1}$ is needed due to the fact that the variance of $d_{t p k}$ is inflated by the factor $\frac{n_{t}+1}{n_{t}}$ when compared to the variance of $\varepsilon_{i j k}$ that is associated with the calibration set.

\section{Example - Noninvasive Measurement of Glucose}

Accurate noninvasive measurement of in vivo glucose levels in diabetics via nearinfrared spectroscopy has proved to be a very difficult task due to the relatively small spectral effect of glucose at physiological levels and the complexity of human tissue. A number of researchers and commercial entities have been heavily involved in this area (see e.g., Heise ${ }^{8}$ ). Arnold et $\mathrm{al}^{9}$ discuss a number of the issues that confront those who attempt to measure glucose noninvasively using multivariate calibration with spectral measurements. The ultimate objective of these activities is to provide a mechanism for diabetic subjects to accurately and conveniently monitor their glucose levels without the pain and inconvenience associated with current monitoring technology that involves measuring the glucose in blood obtained by pricking the skin. The purpose of this example is to demonstrate the feasibility of coupling a generic model with subjectspecific adaptation to provide clinically useful noninvasive measurements of glucose. 
Researchers at Sandia National Laboratories, the University of New Mexico, and Rio Grande Medical Technologies, Inc. have conducted a number of clinical studies associated with the noninvasive measurement of invivo glucose using near-infrared reflectance spectroscopy. One such study is discussed here. To protect the interests of the commercial party involved in this collaboration, many details of the study (which are important but unrelated to the focus of this paper) are not discussed here. Nevertheless, some of the relevant specifics concerning the study can be provided and are as follows.

Calibration data were obtained from 18 diabetic subjects who were repeatedly measured over a span of 7 weeks. The intent of observing the subjects for such a long period of time was to develop calibration data that spanned significant levels of natural intra-subject physiological variation (including but not limited to glucose variation) and sampling variation. In addition, the study protocol involved the deliberate perturbation of the spectrometer and its local environment to induce instrumental/environmental effects into the generic calibration data. These perturbations were carefully selected to span the expected long-term operating conditions of the instrument. Activities, such as these, are extremely important for developing calibration data that will facilitate valid predictions into the future ${ }^{7,11}$.

Spectral and reference data were acquired twice per week from most subjects. A few subjects were unable to keep all of their appointments to provide spectral and reference data. During each appointment, 5 separate spectral measurements at different spatial positions on the underside of the forearm were acquired over a 15-minute period using reflectance sampling from 4200-7200 wavenumbers (390 discrete wavelengths were involved). In addition, two capillary glucose reference measurements were 
obtained via blood draws from each subject during each data acquisition period. The blood draws were performed immediately before and after the acquisition of the spectral data. The spectra, based on the logarithm of the reflected intensities, were not background corrected. Time-based interpolation was used to assign an appropriate capillary glucose reference value to each spectrum. A total of 1161 spectra (other acquired spectra were deemed outliers and were discarded) and associated reference glucose values comprise the calibration data.

The total variation in the spectra within the calibration set is due to a combination of inter- and intra-subject effects. In the case of this example, intra-subject effects include those effects associated with the deliberate perturbation of the spectrometer and its local environment. The total spectral variation was decomposed into intra- and intersubject spectral variation via a wavelength-by-wavelength variance components analysis of the calibration data ${ }^{11}$. Figure 1 displays estimates of the standard deviations of the inter- and intra-subject spectral variation obtained by this analysis. The estimate of intrasubject spectral variation is an aggregate measure obtained across all subjects. Clearly, the inter-subject spectral variation dominates the intra-subject spectral variation. The inter-subject effects were difficult to model in this particular experiment (where the number of subjects was modest) and in many other related experiments where the total number of subjects was much larger. However, the intra-subject effects were found to be consistent in nature across subjects. Thus, a generic modeling approach was deemed appropriate.

In order to test the efficacy of the generic modeling approach, a generic calibration model was developed by using PCR (no intercept) on the spectral and 
capillary glucose reference data that were mean-centered by subject (see Equation 8). The resulting generic model coefficients $\left(\left\{g_{k}\right\}_{\mathrm{k}=1, \ldots, \mathrm{q}}\right)$ are shown in Figure 2. In a qualitative sense, this model corresponds well with the relatively strong glucose bands in the vicinity of $4300 \mathrm{~cm}^{-1}$ and $4400 \mathrm{~cm}^{-1}$. The generic model was then adapted to two additional diabetic subjects who were distinct from the 18 subjects whose data were used to develop the generic calibration data/model. The period of observation for these two additional subjects spanned more than six months, beginning with the initial measurements of the original 18 subjects. Thus, the two additional subjects were observed for more than four months following the acquisition of the generic calibration data. As in the case of acquiring the calibration data, 5 separate spectral measurements at different spatial positions on the underside of the forearm were acquired over a 15-minute period. In addition, capillary glucose reference measurements were acquired from each of the two subjects during each data acquisition period according to the protocol described earlier.

During the first 7 weeks of observation and coinciding with the measurements of the original 18 subjects, the two additional subjects were observed twice per week (with one exception). The additional measurements were made roughly 2 and 4 months beyond the initial 7-week period. The spectra and reference values obtained during the first data acquisition period were used to adapt the generic model to each of the two additional subjects via Equation 12. These adapted models were used to predict the glucose levels associated with subsequently obtained spectra. Figures 3 and 4 compare these predictions (averaged within a data acquisition period) with the reference measurements (also averaged within a data acquisition period) for each subject. The bottom half of each 
figure allows for a direct comparison of predicted glucose with the reference glucose. The top half of each figure provides a visualization of prediction performance versus the measurement sequence. Note that the measurement sequence is not linear in time. The following conventions are used in each figure. The solid lines connect the reference glucose values in the top half of each figure and provide a line of identity in the bottom half of each figure. The " $\mathrm{x}$ " symbol denotes the predictions during the adaptation period (by definition the average prediction is identical to the average reference in this case). The '*' symbols denote predictions made during the remainder of the initial 7 -week period that was spanned by the calibration data. Therefore, these predictions are not prospective with respect to instrument effects. However, the model building process was completely blind with respect to all effects associated with the two additional subjects. The 'o' symbols denote predictions made after the initial 7-week period. These predictions are prospective in every sense (subject and instrument effects) as the model building process was completed immediately following the end of the initial 7 -week period. Again, as in the calibration, the spectra were not background corrected.

It is interesting to note that there is no apparent degradation in prediction performance with respect to the first subject over the 6-month period of observation following tailoring (see Figure 3). In contrast, with respect to the second subject (see Figure 4), prediction performance worsened over time. In this case, the adapted model consistently underpredicted glucose (by about $40 \mathrm{mg} / \mathrm{dL}$ ) over the last several data acquisition periods (perhaps due to some unmodeled physiological effect). Unfortunately, in these particular cases, the outlier metric described in Equation 14 did not indicate a problem. One way to remedy the systematic prediction errors such as those 
observed in the case of the second subject would be to re-adapt the generic model to a subject on a regular basis. This would have the effect of reducing the frequency of painful finger sticks from several times per day to perhaps several times per month or less. Implicitly such a procedure would also provide some benefit with regard to calibration maintenance as the re-adaptation might adjust to a new instrument state that was improperly modeled.

\section{Discussion}

The previous section has provided a single example of the proposed method in a limited context. In fact, adaptation could be thought of in a much broader sense. For example, the adaptation could target a specific geophysical site or measurement environment (e.g., chemical reactor) wherein the analyte of interest is varying over time and where each environment produces a consistent and unique spectral effect. One might want to employ the proposed methodology when the spectral variation across sites or environments is significant and/or difficult to model. Independent of the context, the successful use of generic modeling requires that the intra-object (or e.g., intra-site) spectral effects are similar across objects. Furthermore, it is also imperative that the calibration data that are collected span an appropriate variety of intra-object spectral effects. In this regard, the importance of an effective experimental design cannot be overstated. In the example that was given, a significant amount of thought was given to developing the protocols that were used to acquire the intra-subject spectral data.

The concept of generic modeling might also be profitably extended to calibration transfer, which here refers to the process of migrating a master calibration 
model developed on one or more host spectrometers to a specific target spectrometer. Here, all spectrometers are assumed to be of the same fixed make/model. Due to manufacturing variation across instruments, each instrument will differ in subtle ways such that the same object will appear slightly different across instruments (e.g., resulting in slightly different spectra).

As an example, consider the noninvasive measurement of glucose in the home by a diabetic who is paired with a single instrument. The prospects for developing an effective calibration model based solely on the data from that one diabetic subject would seem to be dubious because of the intensive sampling (associated with that subject) that would be required to develop an operable model. A more effective (and efficient) approach might be to construct a generic calibration model based on intrasubject/instrument spectral variation that is collected from one or more instruments of the same make/model (as was done in the example that was described earlier). If the intrasubject/instrument variation is consistent across instruments, then the series of measurements that are taken to adapt to the subject simultaneously provide adaptation to the specific instrument and current instrument state. Thus, a single generic model could be adaptable to an arbitrary subject being measured on an arbitrary unit from an entire production run of instruments of the same make/model.

At this point the extension to calibration transfer might seem somewhat speculative. However, if one were to pursue this approach, it would be important to identify and replace hardware components that give rise to inconsistent intra-subject effects across instruments of the same make/model. Given a family of instruments with such characteristics, it is also important to develop the generic calibration data over a 
broad set of conditions (using the host instrument(s)) that span the conditions that are expected during the operation of the target instrument(s).

\section{Conclusion}

This paper proposes a new approach for predicting an analyte of interest within and across new objects when it is difficult to model inter-object spectral effects adequately. This approach requires an accompanying experimental protocol that focuses on the acquisition of a sufficiently wide variety of intra-object spectral variation (including that due to variation of the level of the analyte of interest). The proposed approach also requires spectra and associated analyte reference values to adapt to a new object. While not suited for all applications (e.g., where the required reference values are difficult to obtain), this approach could be very useful in a number of applications including the noninvasive measurement of glucose in a home environment. Furthermore, this approach could easily be extended to calibration transfer.

\section{Acknowledgements}

I am grateful to have been a part of the collaboration between Sandia National Laboratories, the University of New Mexico, and Rio Grande Medical Technologies, Inc. that produced the experimental data that was discussed in the illustrative example. I give special thanks to the data acquisition team at RGMT for carefully collecting the experimental data. In addition, I thank Kathy Alam and Mark Benak for a number of suggestions that improved the readability of this manuscript. Sandia is a multiprogram laboratory operated by Sandia Corporation, a Lockheed Martin Company, for the United States Department of Energy under Contract DE-AC04-94AL85000. 


\section{References}

(1) Martens, H.; Naes T., Multivariate Calibration, Wiley, Chichester, 1989.

(2) Naes T., Technometrics, 1985, 27, 301-311.

(3) Franke, J. E.; Niemcyzk, T. M.; Haaland, D. M. Spectrochemica Acta. 1994, 50A, 168-1723.

(4) Heise, H. M. Hormone and Metabolic Research 1996, 28, 527-534.

(5) Gottfries, J.; Depui, H.; Fransson, M.; Jongeneelen, M.; Josefson, M.; Langkilde, F. W.; Witte, D. T. Journal of Pharmaceutical and Biomedical Analysis 1996, 14, 1495-1503.

(6) Lavine, B. K. Anal. Chem. 1998, 70, 209R-228R.

(7) Thomas, E. V.; Ge N. Technometrics 2000, 42 (In Press).

(8) Heise, H. M. "Near-infrared Spectrometry for in vivo Glucose Sensing" in Biosensors in the Body: Continuous in vivo Monitoring (D. M. Fraser, editor), John Wiley, 1997.

(9) Arnold, M. A.; Burmeister, J. J.; Small G. W. Anal. Chem. 1998, 70, 1773-1782.

(10) Swierenga, H.; de Weijer, A. P.; van Wink, R. J.; Buydens, L. M. C., Chemometrics and Intelligent Laboratory Systems 1999, 49, 1-17.

(11) Searle, S. R; Casella, G.; McColloch, C. E., Variance Components, Wiley, New York, 1992. 


\section{FIGURE CAPTIONS}

Figure 1 - Standard Deviation of Inter-Subject and Intra-Subject Spectral Variation. Figure 2 - Generic Model Coefficients.

Figure 3 - Generic Model Performance: Subject 1, Predicted Glucose versus Reference Glucose. Solid line connects reference glucose values, ' $x$ ' denotes prediction during adaptation period, '*' denotes prediction during remainder of initial 7-week period, and ' $o$ ' denotes prediction following initial 7-week period.

Figure 4 - Generic Model Performance: Subject 2, Predicted Glucose versus Reference Glucose. Solid line connects reference glucose values, ' $x$ ' denotes prediction during adaptation period, '*' denotes prediction during remainder of initial 7-week period, and 'o' denotes prediction following initial 7-week period. 
Figure 1

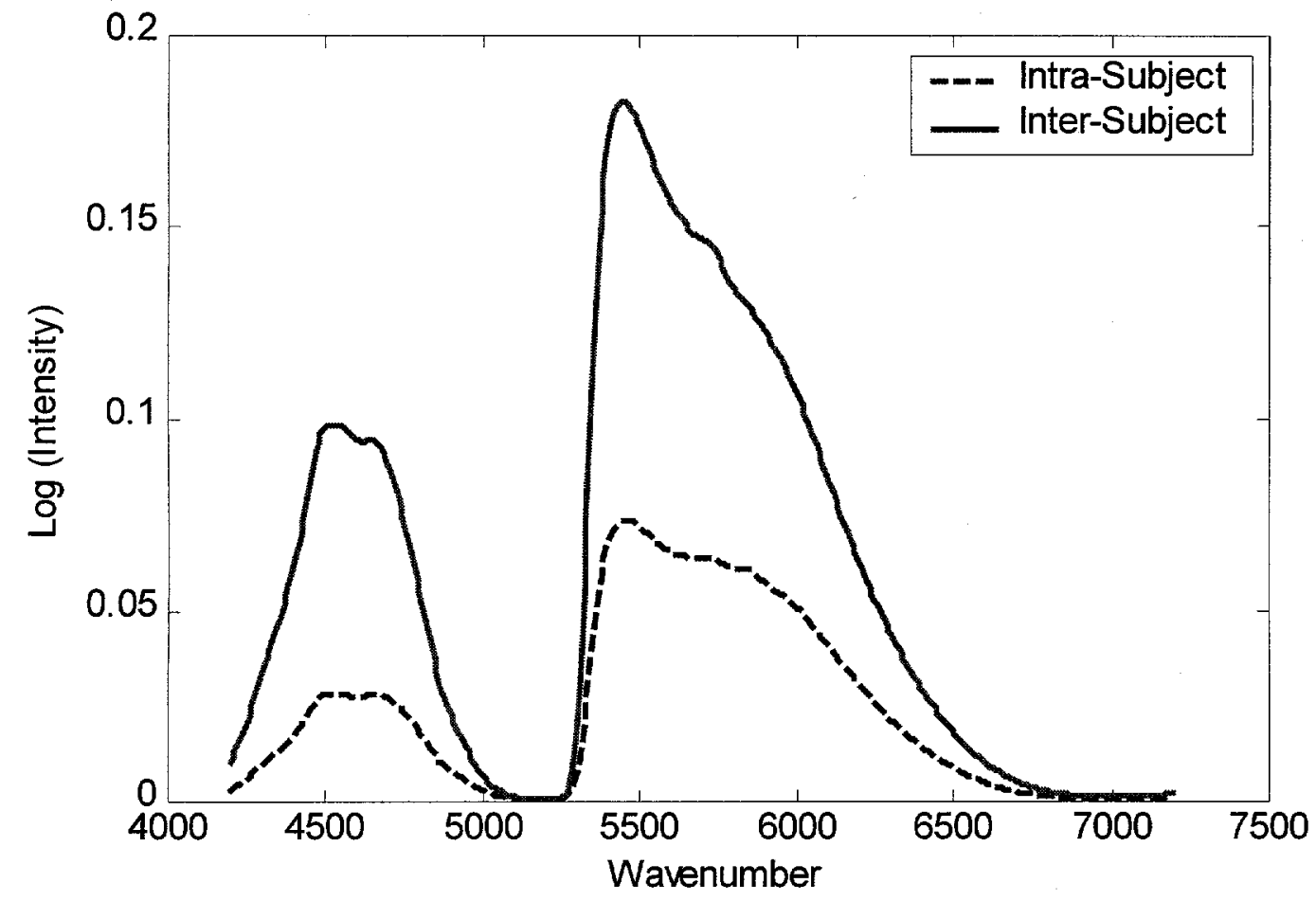


Figure 2

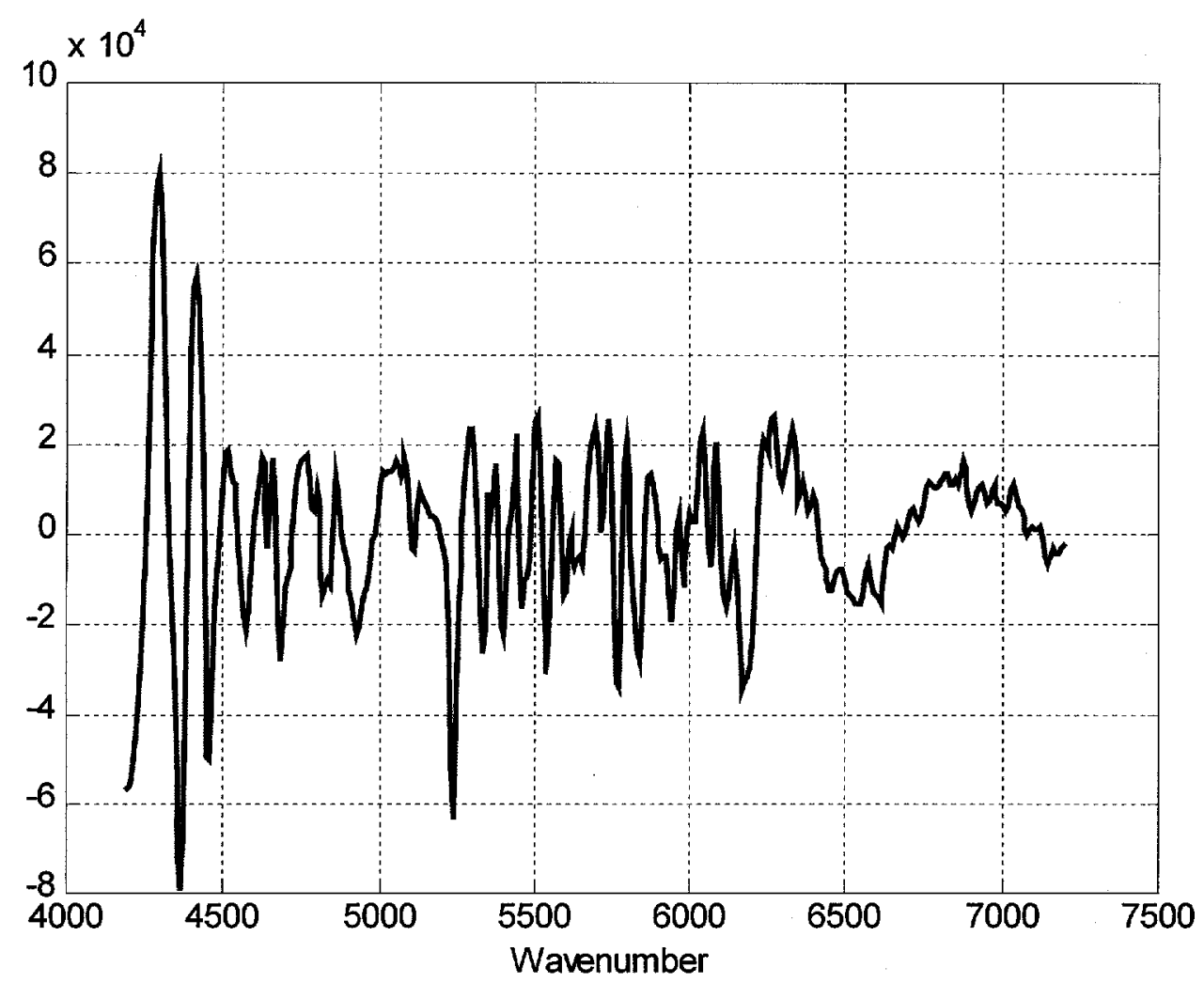


Figure 3
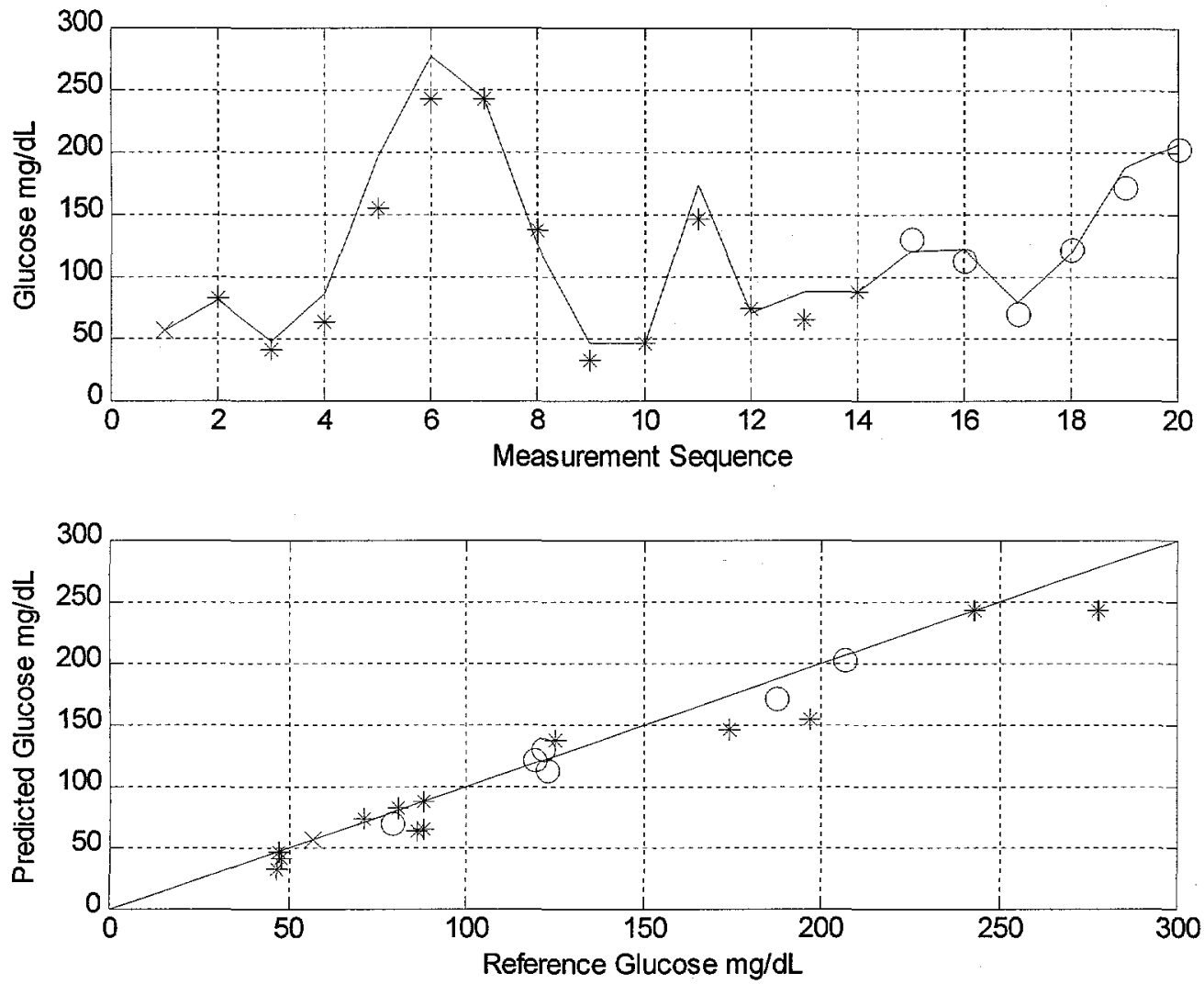
Figure 4
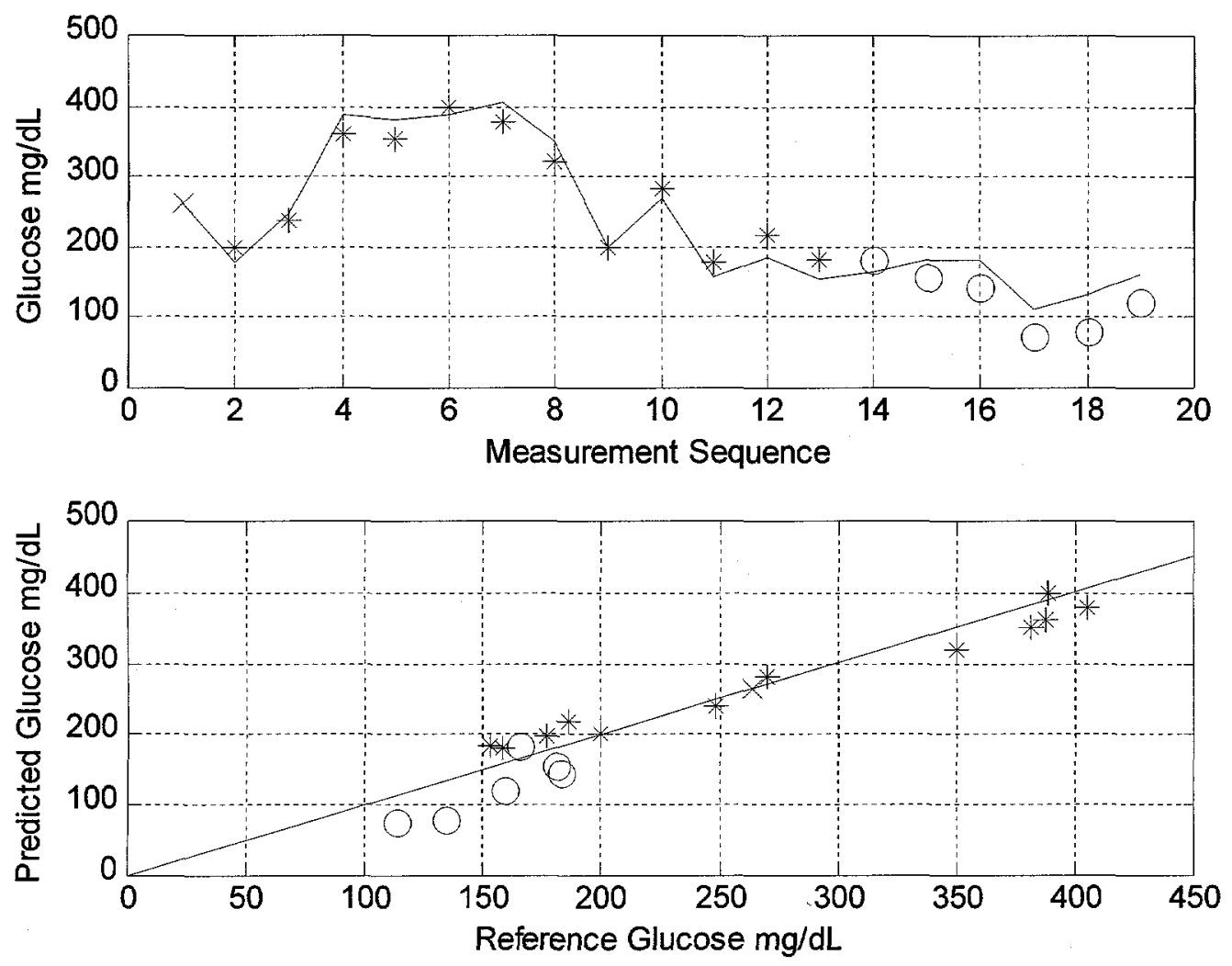